\title{
Algorithmic Procedures for Constructing Ontological Representations of Production Technologies
}

\author{
Timur Mironov \\ Institute of Engineering Sciences \\ Pskov State University \\ Pskov, Russia \\ jarwis9812@gmail.com \\ Lilia Motaylenko \\ Institute of Engineering Sciences \\ Pskov State University \\ Pskov, Russia \\ _lvs_@mail.ru
}

\author{
Dmitry Andreev \\ Institute of Engineering Sciences \\ Pskov State University \\ Pskov, Russia \\ dandreev60@mail.ru \\ Elena Lineva \\ Institute of Engineering Sciences \\ Pskov State University \\ Pskov, Russia \\ elenalinewa@yandex.ru
}

\author{
Iuliia Bruttan \\ Institute of Engineering Sciences \\ Pskov State University \\ Pskov, Russia \\ bruttan@mail.ru
}

\begin{abstract}
This paper focuses on formalized description of technologies as a category of procedural knowledge. It describes the model of ontological representation of technologies. The authors present the algorithms of staged combined design of unified decomposition constructions that enable to form decomposition structures of technologies. The article introduces the extended algorithm to construct ontological representation of technologies.
\end{abstract}

Keywords - algorithm, ontology, technology, unified decomposition construction.

\section{INTRODUCTION}

Modern researchers are increasingly focusing on the study of issues connected with improving approaches to knowledge representation in the field of systems analysis and design. Modern information space is being enriched rapidly with procedural knowledge that people gain in the process of life exploring new procedures, methods, ways of actions, getting new knowledge and exchanging it with other people in the process of cooperative activities and communication. Technological knowledge is mostly procedural as its intended purpose is substantive and practical work of every person aimed at transforming the surrounding reality [1].

In this respect, it is quite natural that the interest of some researchers in the construction of the ontology of processes has significantly increased over the past decades. However, some of them argue the fact that real methods of developing the ontology of processes exist [2]. Main areas of scientists' activities are given in works [3] [17], which represent the most notable results in the field of ontological engineering of processes. It seems preferable to use multilevel ontological systems, computer-aided design systems and ontological systems with active semantics solving the problems connected with arrangement of formalized presentation of technological knowledge. At the same time, the cumulative disadvantage of these systems is that formal mechanisms of the technologies' description at the applied level are poorly elaborated. The problem is the absence of actions' consideration as holistic formations, the sets of participants in which could determine the relations between the corresponding concepts. Also, the problem is accompanied with the replacement of the axiomatics of ontological models with modern graphical tools for arranging and presenting knowledge.

This article focuses on algorithmic procedures that lay the foundation for the implementation of the software OntoTechnology [18], that is meant to be used for formalized description of technologies. These algorithms implement the logical procedures for automated and automatic construction of ontological representations of technologies [19]. Among them are the technologies of clothing production [20], a significant part of construction technologies [21] and mechanical engineering technologies [22].

Online ISSN 2256-070X

https://doi.org/10.17770/etr2021vol2.6624 (C) 2021 Timur Mironov, Dmitry Andreev, Iuliia Bruttan, Lilia Motaylenko, Elena Lineva. Published by Rezekne Academy of Technologies. This is an open access article under the Creative Commons Attribution 4.0 International License 


\section{MATERIALS AND METHODS}

The author in work [23] represents by a tuple the model of ontological representations of technologies according to which the ordered set of unified decomposition constructions (UDC) forms decomposition structure of technology (DST):

$$
\begin{gathered}
\text { Ont }_{\text {Tech }}=<T D, P, F>\text {, (1) } \\
\text { where } T D=\bigcup_{u} T D_{u}-\text { a set of concepts of }
\end{gathered}
$$

technological actions; $P$ - intra-level relation of immediate precedence that is used to describe the relations between fully formed private concepts of one level of decomposition UDC; $F$ - inter-level relation of "partwhole" that is used to describe the relations between every fully formed private concepts and the already fully formed holistic concept UDC.

The model of the concept of technological action that is the basic representation of every technological action located in the nodes of DST, is represented by a tuple [24]:

$$
T D_{u}=<T P_{u}, Y_{u}, X_{u} \mid W_{u}, H_{u}, Z_{u}>\text {, }
$$

where $T D_{u}$ - a concept of technological action, - a kernel of the concept, $Y_{u}$ - a set of resulting components, $X_{u}$ - a set of source components, $W_{u}$ - a set of invariant components, $H_{u}$ - a set of cost characteristics, $Z_{u}$ - a set of own characteristics.

The process of constructing a formalized description of technologies is to implement the theoretical propositions formulated and proved in work [23]. They make DST formation possible by means of staged combined design of UDC starting with the root representations of technologies.

The implementation of main stages of UDC construction goes in accordance with the execution of the following algorithmic procedures (dotted line in "Fig. 1"):

1. The algorithm for the automated construction of decomposition of a preliminary formed concept (stage of downward design);

2. The algorithm for the automatic determination of relations between fully formed private concepts (stage of intra-level design);

3. The algorithm for the automatic determination of the complete formation of a holistic concept (stage of upward design).
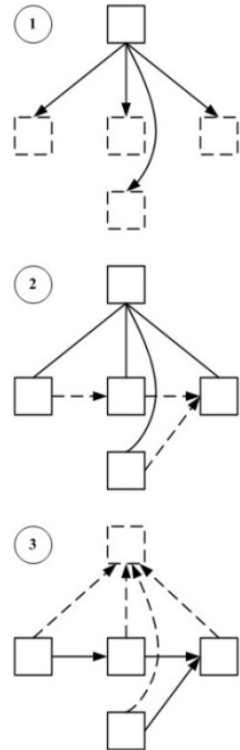

Fig. 1. Staged algorithmic procedures for constructing UDC

Further, the process of construction UDC will be described using the example of UDC at the apex with the concept $\lambda$ DST level. Main patterns of this process' implementation are given in works [25] - [27].

\section{RESULTS AND DISCUSSION}

The algorithm for the automated construction of decomposition of a preliminary formed concept

The algorithm is given in "Fig. 2". Block 2 performs the initial initialization of each set determining the structure of the concept $T D_{\alpha_{i}, \beta_{j}, \ldots, \eta_{l}, \lambda_{m}}$ by empty sets. Then the elements of the kernel $T P_{\alpha_{i}, \beta_{j}, \ldots, \eta_{l}, \lambda_{m}}$ and set $Y_{\alpha_{i}, \beta_{j}, \ldots, \eta_{l}, \lambda_{m}}$ of the concept $T D_{\alpha_{i}, \beta_{j}, \ldots, \eta_{l}, \lambda_{m}}$ are manually entered. After that, the verification of the fact of their cumulative non-emptiness is implemented. If this condition is true, then a message is displayed stating that “Concept $T D_{\alpha_{i}, \beta_{j}, \ldots, \eta_{l}, \lambda_{m}}^{\prime}$ is preliminary formed". Otherwise, a message is displayed stating that "Concept $T D_{\alpha_{i}, \beta_{j}, \ldots, \eta_{l}, \lambda_{m}}$ is not preliminary formed" and then we come back to manual entry stage. 
Environment. Technology. Resources. Rezekne, Latvia Proceedings of the $13^{\text {th }}$ International Scientific and Practical Conference. Volume 2, 116-123

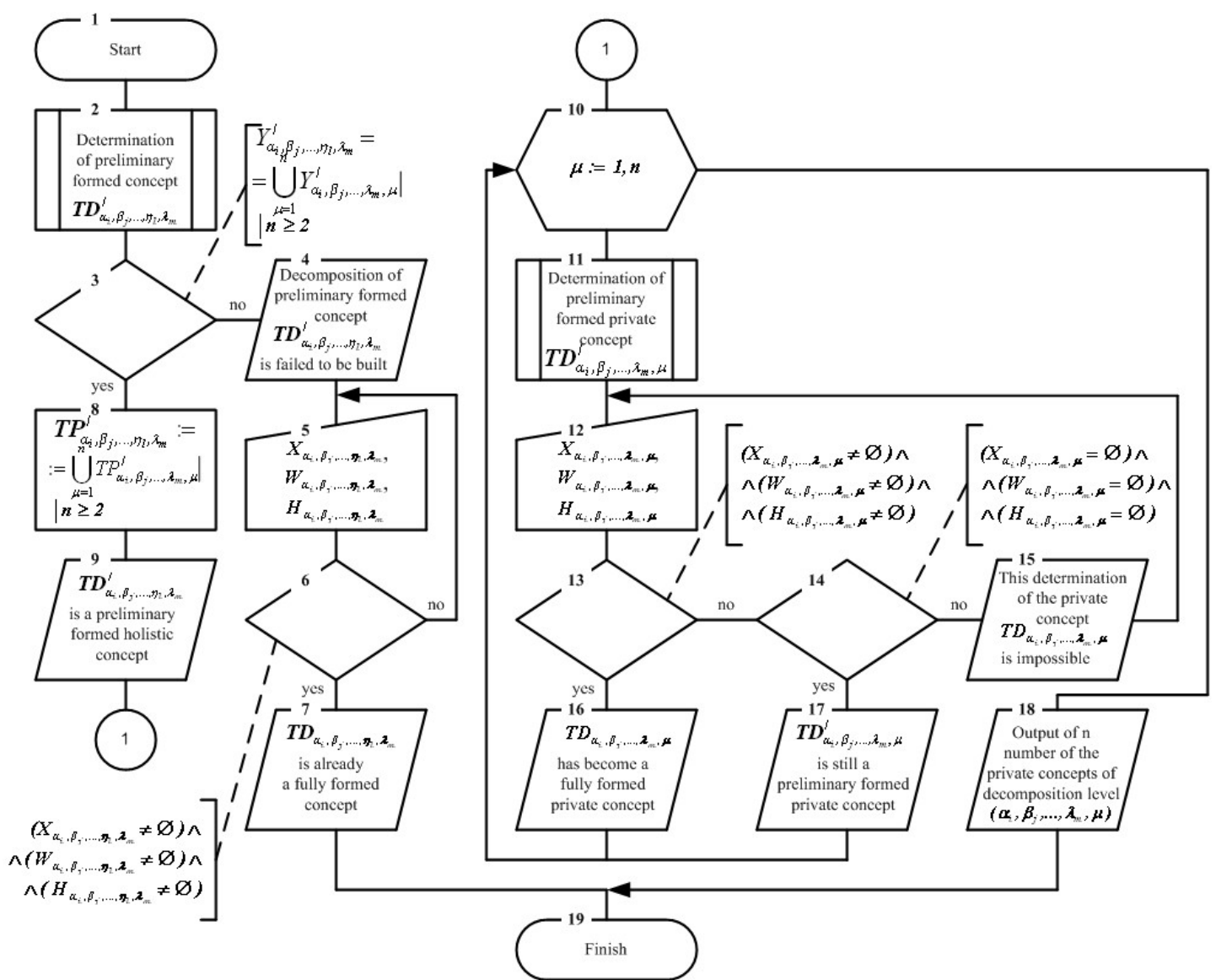

Fig. 2. Block-diagram of the algorithm for the automated construction of decomposition of a preliminary formed concept

Block 3 performs the verification of the determination of set $Y_{\alpha_{i}, \beta_{j}, \ldots, \eta_{l}, \lambda_{m}}^{\prime}$ of preliminary formed concept $T D_{\alpha_{i}, \beta_{j}, \ldots, \eta_{l}, \lambda_{m}}^{\prime}$ as a collection of sets $\bigcup_{\mu=1}^{n} Y_{\alpha_{i}, \beta_{j}, \ldots, \lambda_{m}, \mu}^{\prime}$ if $n \geq 2$. If this condition is not true, then a message is displayed stating that "Decomposition of preliminary formed concept $T D_{\alpha_{i}, \beta_{j}, \ldots, \eta_{l}, \lambda_{m}}^{\prime}$ is failed to be built” (block 4). After that, the elements of the sets $X_{\alpha_{i}, \beta_{j}, \ldots, \eta_{l}, \lambda_{m}}, W_{\alpha_{i}, \beta_{j}, \ldots, \eta_{l}, \lambda_{m}}$ and $H_{\alpha_{i}, \beta_{j}, \ldots, \eta_{l}, \lambda_{m}}$ of concept $T D_{\alpha_{i}, \beta_{j}, \ldots, \eta_{l}, \lambda_{m}}$ are offered to be entered manually in block 5 . Then the verification of the fact of their cumulative non-emptiness is implemented in block 6. If this condition is true, then a message is displayed stating that "Concept $T D_{\alpha_{i}, \beta_{j}, \ldots, \eta_{l}, \lambda_{m}}$ is already fully formed" (block 7) and there is a transition directly to block 19 at the end of the algorithm. Otherwise, we come back to block 5 . If the condition from block 3 is true, then in block 8 the kernel $T P_{\alpha_{i}, \beta_{j}, \ldots, \eta_{l}, \lambda_{m}}^{\prime}$ is determined as a collection of sets of kernels $\bigcup_{\mu=1}^{n} T P_{\alpha_{i}, \beta_{j}, \ldots, \lambda_{m}, \mu}^{\prime}$ if $n \geq 2$ (axiomatic property 3) [23]. After that, block 9 displays a message stating that “Concept $T D_{\alpha_{i}, \beta_{j}, \ldots, \eta_{l}, \lambda_{m}}^{\prime}$ is a preliminary formed holistic concept".

Block 10 illustrates how to loop through each of the concepts $\bigcup_{\mu=1}^{n} T D_{\alpha_{i}, \beta_{j}, \ldots, \lambda_{m}, \mu}$. Block 11 performs the initial initialization of each set determining the structure of the concept $T D_{\alpha_{i}, \beta_{j}, \ldots, \lambda_{m}, \mu}$ by empty sets. Then the elements of the kernel $T P_{\alpha_{i}, \beta_{j}, \ldots, \lambda_{m}, \mu}$ and set $Y_{\alpha_{i}, \beta_{j}, \ldots, \lambda_{m}, \mu}$ of the concept $T D_{\alpha_{i}, \beta_{j}, \ldots, \lambda_{m}, \mu}$ are manually entered. After that, the verification of the fact of their cumulative non-emptiness is implemented. If this condition is true, then a message is displayed stating that "Concept $T D_{\alpha_{i}, \beta_{j}, \ldots, \lambda_{m}, \mu}^{\prime}$ is a preliminary formed private concept”. Otherwise, a message is displayed stating that "Concept $T D_{\alpha_{i}, \beta_{j}, \ldots, \lambda_{m}, \mu}$ is not a preliminary formed private concept" and then we come back to manual entry stage. After that, the elements of the sets $X_{\alpha_{i}, \beta_{j}, \ldots, \lambda_{m}, \mu}, \quad W_{\alpha_{i}, \beta_{j}, \ldots, \lambda_{m}, \mu}$ and 
$H_{\alpha_{i}, \beta_{j}, \ldots, \lambda_{m}, \mu}$ of concept $T D_{\alpha_{i}, \beta_{j}, \ldots, \lambda_{m}, \mu}$ are offered to be entered manually in block 12 . Then the verification of the fact of their cumulative non-emptiness is implemented in block 13. If this condition is true, then a message is displayed stating that "Concept $T D_{\alpha_{i}, \beta_{j}, \ldots, \lambda_{m}, \mu}$ has become a fully formed private concept" (block 16) and there is a transition directly to block 10 to the next concept. Otherwise, the verification of the fact of cumulative emptiness of sets $X_{\alpha_{i}, \beta_{j}, \ldots, \lambda_{m}, \mu}$ , $W_{\alpha_{i}, \beta_{j}, \ldots, \lambda_{m}, \mu}$ and $H_{\alpha_{i}, \beta_{j}, \ldots, \lambda_{m}, \mu}$ of concept $T D_{\alpha_{i}, \beta_{j}, \ldots, \lambda_{m}, \mu}$ is implemented in block 14. If this condition is true, then a message is displayed stating that "Concept $T D_{\alpha_{i}, \beta_{j}, \ldots, \lambda_{m}, \mu}^{\prime}$ is still a preliminary formed private concept" (block 17) and there is a transition directly to block 10 to the next concept. Otherwise, a message is displayed stating that "This determination of the private concept $T D_{\alpha_{i}, \beta_{j}, \ldots, \lambda_{m}, \mu}$ is impossible" (block 15) and there is a transition directly to block 12. After looping through each of the private concepts $\bigcup_{\mu=1}^{n} T D_{\alpha_{i}, \beta_{j}, \ldots, \lambda_{m}, \mu}$, their number is displayed $n$ (block 18). Then there is a transition directly to block 19 at the end of the algorithm.

The algorithm for the automatic determination of relations between fully formed private concepts

This algorithm is given in "Fig. 3". Block 2 illustrates how to loop through each of the private concepts $\bigcup_{\mu=1}^{n} T D_{\alpha_{i}, \beta_{j}, \ldots, \lambda_{m}, \mu}$.

Block 3 performs verification of the fact either certain private concept $T D_{\alpha_{i}, \beta_{j}, \ldots, \lambda_{m}, \mu}$ is fully formed. If this condition is true, a message is displayed stating that "Concept $T D_{\alpha_{i}, \beta_{j}, \ldots, \lambda_{m}, \mu}$ is a fully formed private concept" (block 6). Otherwise, a message is displayed stating that "Concept $T D_{\alpha_{i}, \beta_{j}, \ldots, \lambda_{m}, \mu}^{\prime}$ is a preliminary formed private concept" (block 4). After that, a message is displayed stating that "It is impossible to establish a relation $P$ for preliminary formed private concept $T D_{\alpha_{i}, \beta_{j}, \ldots, \lambda_{m}, \mu}^{\prime}$ ” (block 5) and there is a transition back to block 2 to the next concept.
Block 7 checks the existence of this fully formed private concept $T D_{\alpha_{i}, \beta_{j}, \ldots, \lambda_{m}, \mu_{s}}$ for certain fully formed private concept $T D_{\alpha_{i}, \beta_{j}, \ldots, \lambda_{m}, \mu}$ to fulfill the condition that $Y_{\alpha_{i}, \beta_{j}, \ldots, \lambda_{m}, \mu}=X_{\alpha_{i}, \beta_{j}, \ldots, \lambda_{m}, \mu_{s}}$. If this condition is true, a message is displayed stating that "There is only one such fully formed private concept $T D_{\alpha_{i}, \beta_{j}, \ldots, \lambda_{m}, \mu}$, which has a relation $P$ with fully formed private concept $T D_{\alpha_{i}, \beta_{j}, \ldots, \lambda_{m}, \mu_{s}}$.

Also, there is a graphical drawing of the relation $P$ from fully formed private concept $T D_{\alpha_{i}, \beta_{j}, \ldots, \lambda_{m}, \mu}$ to fully formed private concept $T D_{\alpha_{i}, \beta_{j}, \ldots, \lambda_{m}, \mu_{s}}$ (block 8), and there is a transition back to block 2 to the next concept. Otherwise, block 9 checks the existence of this fully formed private concept $T D_{\alpha_{i}, \beta_{j}, \ldots, \lambda_{m}, \mu_{s}}$ for certain fully formed private concept $T D_{\alpha_{i}, \beta_{j}, \ldots, \lambda_{m}, \mu}$ to fulfill the condition that $Y_{\alpha_{i}, \beta_{j}, \ldots, \lambda_{m}, \mu} \subset X_{\alpha_{i}, \beta_{j}, \ldots, \lambda_{m}, \mu_{s}}$. If this condition is false, a message is displayed stating that "This fully formed private concept $T D_{\alpha_{i}, \beta_{j}, \ldots, \lambda_{m}, \mu_{s}}$, there is a relation between $P$ and certain fully formed private concept $T D_{\alpha_{i}, \beta_{j}, \ldots, \lambda_{m}, \mu}$ does not exist” (block 10 ), and there is a transition back to block 2 to the next concept. Otherwise, block 11 checks the existence of the set of these fully formed private concepts $\bigcup_{k=1}^{n^{\prime}} T D_{\alpha_{i}, \beta_{j}, \ldots, \lambda_{m}, \mu_{v_{k}}}$ for certain fully formed private concept $T D_{\alpha_{i}, \beta_{j}, \ldots, \lambda_{m}, \mu}$ to fulfill the condition that $\bigcup_{k=1}^{n^{\prime}} Y_{\alpha_{i}, \beta_{j}, \ldots, \lambda_{m}, \mu_{v k}} \subseteq\left(X_{\alpha_{i}, \beta_{j}, \ldots, \lambda_{m}, \mu_{s}} \mid X_{\alpha_{i}, \beta_{j}, \ldots, \lambda_{m}, \mu_{s}}^{*}\right)$. If this condition is true, there is a graphical relation $P$ from fully formed privawing of the $T D_{\alpha_{i}, \beta_{j}, \ldots, \lambda_{m}, \mu}$ to fully formed private concept $T D_{\alpha_{i}, \beta_{j}, \ldots, \lambda_{m}, \mu_{s}}$ (block 12), and there is a transition back to block 2 to the next concept. Otherwise, and there is a transition back to block 8, and then back to block 2 to the next concept. After looping through each of the private concepts $\bigcup_{\mu=1}^{n} T D_{\alpha_{i}, \beta_{j}, \ldots, \lambda_{m}, \mu}$ (block 2) there is a transition back to block 13 at the end of the algorithm. 
Environment. Technology. Resources. Rezekne, Latvia Proceedings of the $13^{\text {th }}$ International Scientific and Practical Conference. Volume 2, 116-123

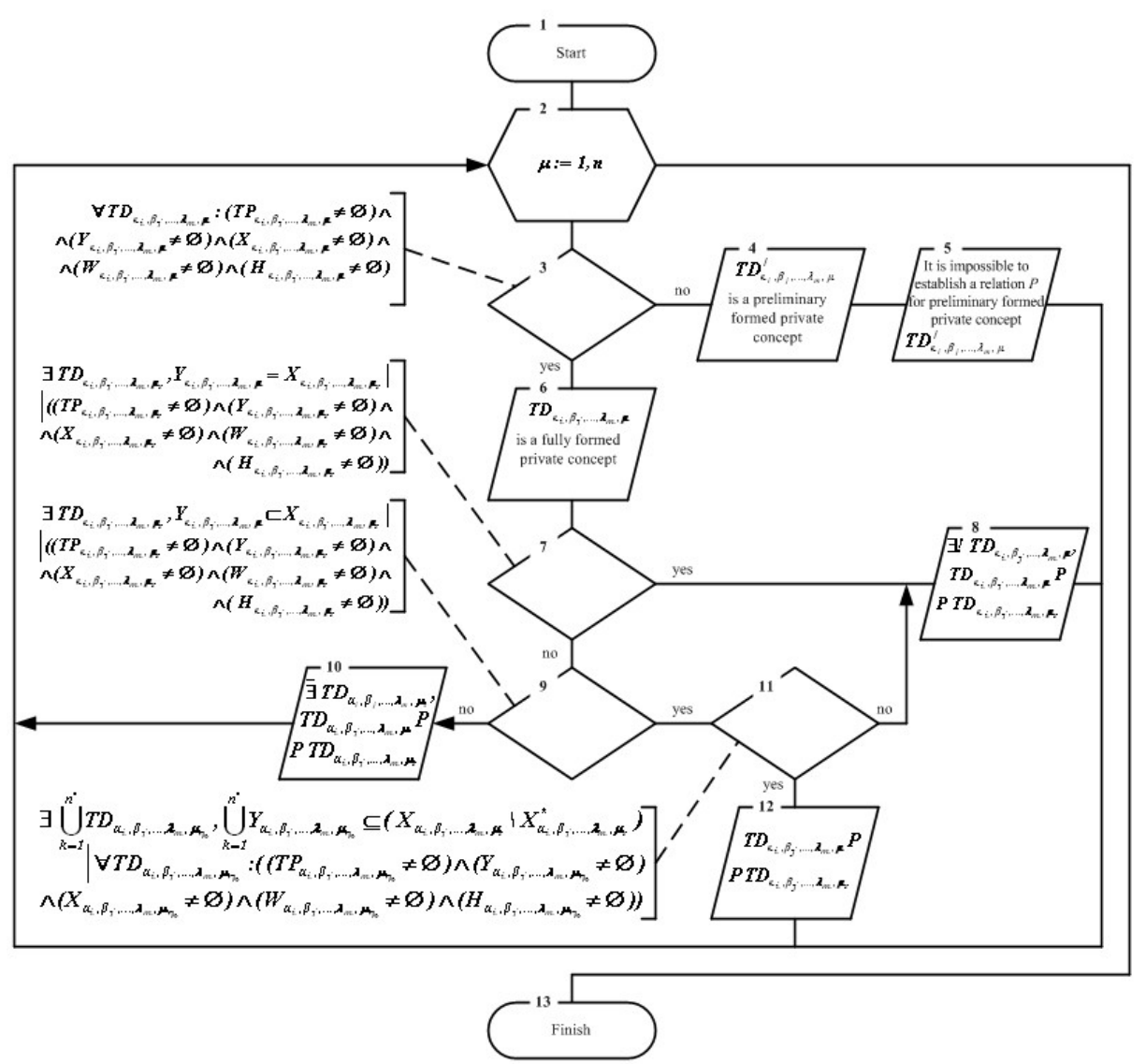

Fig. 3. Block-diagram of the algorithm for the automatic determination of relations between fully formed private concepts

The algorithm for the automatic determination of the complete formation of a holistic concept

This algorithm is given in "Fig. 4". Block 2 illustrates how to loop through each of the private concepts $\bigcup_{\mu=1}^{n} T D_{\alpha_{i}, \beta_{j}, \ldots, \lambda_{m}, \mu}$. Block 3 checks the existence of this fully formed private concept $T D_{\alpha_{i}, \beta_{j}, \ldots, \lambda_{m}, \mu_{q}}$ for certain fully formed private concept $T D_{\alpha_{i}, \beta_{j}, \ldots, \lambda_{m}, \mu}$ to fulfill the condition that $Y_{\alpha_{i}, \beta_{j}, \ldots, \lambda_{m}, \mu_{q}}=X_{\alpha_{i}, \beta_{j}, \ldots, \lambda_{m}, \mu}$. If this condition is true, set $X_{\alpha_{i}, \beta_{j}, \ldots, \eta_{l}, \lambda_{m}}$ of a holistic concept $T D_{\alpha_{i}, \beta_{j}, \ldots, \eta_{l}, \lambda_{m}}$ is the same (block 4) and there is a transition to block 8 . If the condition from block 3 is not fulfilled, block 5 checks the existence of this fully formed private concept $T D_{\alpha_{i}, \beta_{j}, \ldots, \lambda_{m}, \mu_{q}}$ for certain fully formed private concept $T D_{\alpha_{i}, \beta_{j}, \ldots, \lambda_{m}, \mu}$ to fulfill the condition that $Y_{\alpha_{i}, \beta_{j}, \ldots, \lambda_{m}, \mu_{q}} \subset X_{\alpha_{i}, \beta_{j}, \ldots, \lambda_{m}, \mu}$. If this condition is true, the set $X_{\alpha_{i}, \beta_{j}, \ldots, \eta_{l}, \lambda_{m}}$ of the holistic concept $T D_{\alpha_{i}, \beta_{j}, \ldots, \eta_{l}, \lambda_{m}}$ is replenished with a complement $\mid X_{\alpha_{i}, \beta_{j}, \ldots, \lambda_{m}, \mu}^{*}$ of a set $X_{\alpha_{i}, \beta_{j}, \ldots, \lambda_{m}, \mu}$ of a fully formed private concept $T D_{\alpha_{i}, \beta_{j}, \ldots, \lambda_{m}, \mu}$ (block 6) and there is a transition to block 8 . If the condition from block 5 is not true, the set $X_{\alpha_{i}, \beta_{j}, \ldots, \eta_{l}, \lambda_{m}}$ of the holistic concept $T D_{\alpha_{i}, \beta_{j}, \ldots, \eta_{l}, \lambda_{m}}$ is replenished with a set $X_{\alpha_{i}, \beta_{j}, \ldots, \lambda_{m}, \mu}$ of a certain fully formed private concept $T D_{\alpha_{i}, \beta_{j}, \ldots, \lambda_{m}, \mu}$ (block 7) and there is a transition to block 8.

In blocks 8-9 there is a determination of sets $W_{\alpha_{i}, \beta_{j}, \ldots, \eta_{l}, \lambda_{m}}$ and $H_{\alpha_{i}, \beta_{j}, \ldots, \eta_{l}, \lambda_{m}}$ of the holistic concept $T D_{\alpha_{i}, \beta_{j}, \ldots, \eta_{l}, \lambda_{m}}$ (in accordance with axiomatic properties 7 и 8) [23]. After that, there is a transition back to block 2 to the next concept. After looping through all fully formed private concepts $\bigcup_{\mu=1}^{n} T D_{\alpha_{i}, \beta_{j}, \ldots, \lambda_{m}, \mu}$ (block 2), the message is displayed stating that "A fully formed holistic concept $T D_{\alpha_{i}, \beta_{j}, \ldots, \eta_{l}, \lambda_{m}}$ is obtained” (block 10). Looping through all fully formed private concepts $\bigcup_{\mu=1}^{n} T D_{\alpha_{i}, \beta_{j}, \ldots, \lambda_{m}, \mu}$ is organized again (block 11) with the aim of graphical drawing of the relation $F$ from 
each fully formed private concept $T D_{\alpha_{i}, \beta_{j}, \ldots, \lambda_{m}, \mu}$ to the already fully formed holistic concept $T D_{\alpha_{i}, \beta_{j}, \ldots, \eta_{l}, \lambda_{m}}$ (block 12). When the process is finished, there is a transition to block 13 at the end of the algorithm.

It is significant to note that the information about every sets' objects of the source components of fully formed private concepts $\bigcup_{\mu} T D_{\alpha_{i}, \beta_{j}, \ldots, \lambda_{m}, \mu}$, taking part in formation of set $X_{\alpha_{i}, \beta_{j}, \ldots, \eta_{l}, \lambda_{m}}$ of fully formed holistic concept $T D_{\alpha_{i}, \beta_{j}, \ldots, \eta_{l}, \lambda_{m}}$ can be kept in specialized domain ontologies grouped by raw materials. Model of each ontology of this kind $O n t_{O b j}$, can be determined as the following tuple [28]:

$$
O n t_{O b j}=<D_{O b j}, E_{O b j}, G_{O b j}>\text {, }
$$

where $D_{O b j}$ - set of objects' concepts; $E_{O b j}-$ set of properties of objects' concepts; $G_{O b j}$ - "genusspecies” relation with multiple inheritance.

Thus, the systematization of the concepts of the sets' objects of the source components of fully formed concepts $T D$ by means of automated methods constructing of domain ontologies allows to make the "repair" of DST in terms of adjusting the subject orientation of fully formed concepts TD [29].

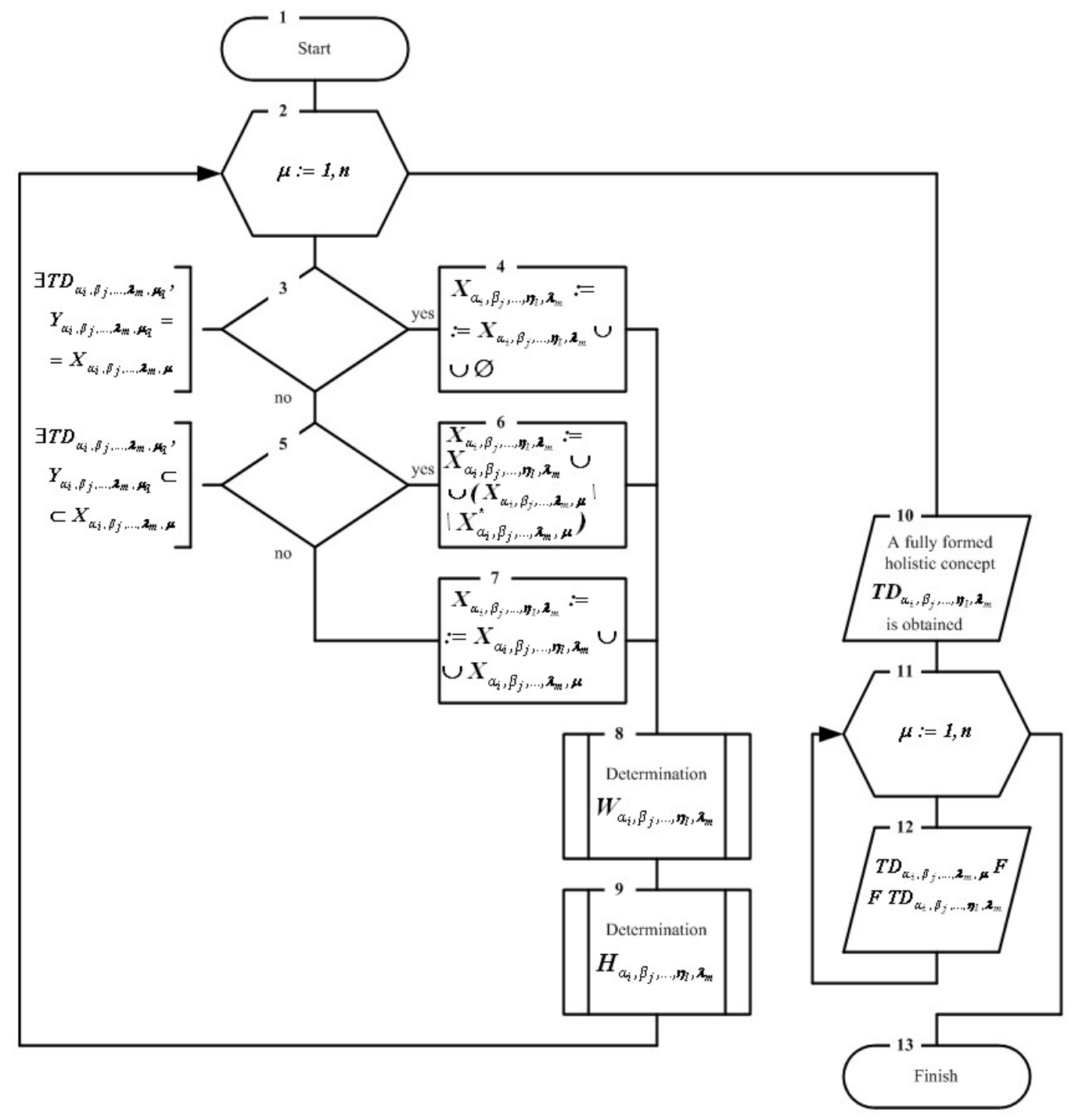

Fig. 4. Block-diagram of the algorithm for the automatic determination of the complete formation of a holistic concept

Extended algorithm for the construction of the formalized description of technologies

This algorithm is given in “Fig. 5". Block 2 performs the initial initialization of some positions in index sequence of root concept DST. Blocks 3-4 give the illustration of stages of downward and intra-level design of UDC. Then, the values of index sequences of preliminary formed concepts of this decomposition level are identified (block 5). Block 6 checks either all concepts of this decomposition level are fully formed. If 
this condition is not true, there is a fixation of a preliminary formed private concept with the already incremented number of positions in index sequence (block 7) with the transition back to block 3. In case the condition of block 6 is true, stage of upward design of UDC is implemented (block 8). After that, there is a fixation in block 9 of a fully formed holistic concept with the already decremented number of positions in

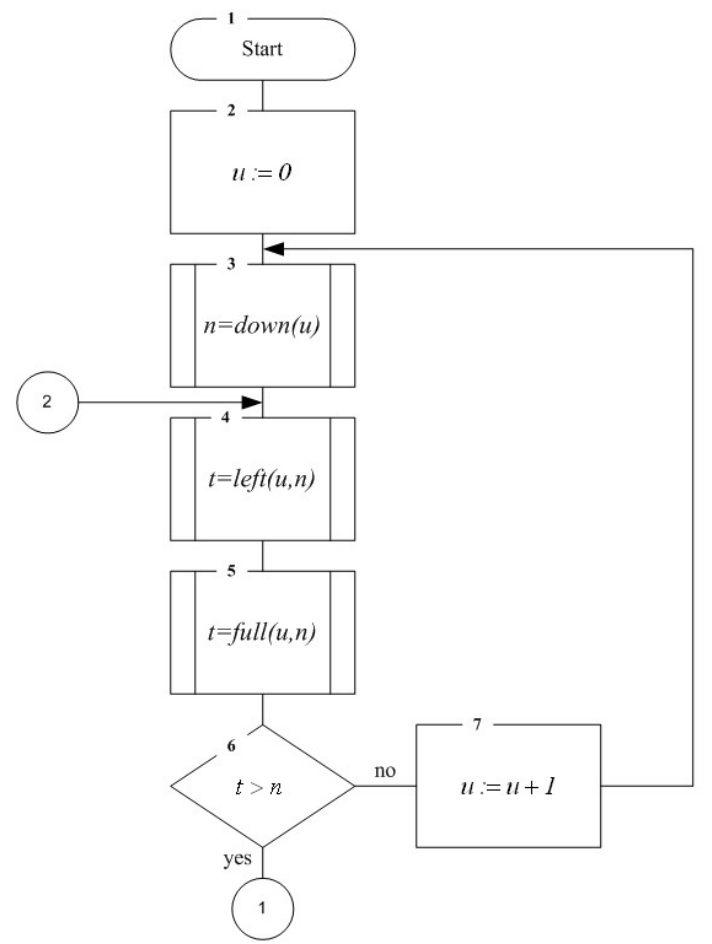

Fig. 5. The extended algorithm for the construction of the formalized description of technologies

\section{CONCLUSION}

This article describes original algorithms of construction of formalized description of technologies that are different from existing ones as they constructing ontological hierarchies of concepts based on predetermined decomposition features, automatic establishment of relations between concepts of the same decomposition level and automatic receiving full information about concepts located in root nodes of DST based on the implemented principle of level-by-level knowledge aggregation.

The consideration of the models' peculiarities and algorithms that are constructed lets us conclude that the method of formalized description of technologies is developed. This method is different from existing ones as it gives a possibility to form DST based on staged combined design of UDC with the aim of structuring the representation of knowledge about technologies with varying degrees of details [23].

The developed models and algorithms of formalized description of technologies and the software OntoTechnology [30] that has proved its efficiency can serve as a basis for creating tools for computer operation of technological knowledge in order to improve the index sequence. Block 10 verifies either the root concept of UDC is reached. If this condition is not true, there is a transition to block 4. This way, if the condition of block 10 is true, a message is displayed stating that "The formalized description of the technology is BUILT!" (block 11) and there is a transition to block 12 at the end of the algorithm.

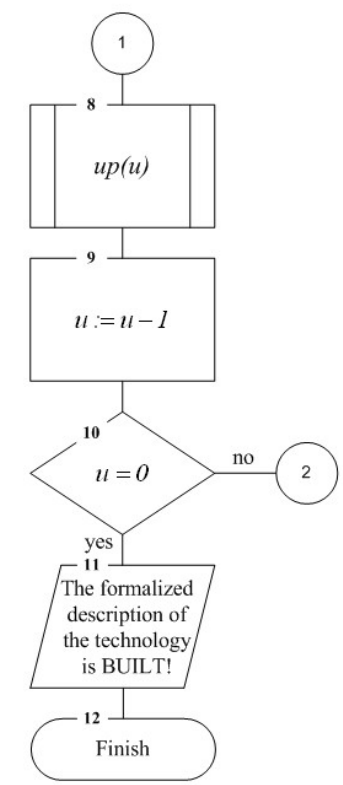

efficiency of solving a wide range of practical problems: constructing specialized stores for describing technologies, selecting the most suitable technologies, analyzing expert studies of technologies, developing educational and methodological and training complexes, and also can become the basis for supporting the processes of technology synthesis.

\section{REFERENCES}

[1] N. N. Shumilkin, "Methodology of technological knowledge," Theory and practice of social development, No. 2, pp. 161-167, 2011. (in Russian)

[2] A. V. Palagin and N. G. Petrenko, "System-ontological analysis of the subject area," Control systems and machines, No. 4, pp. 314, 2009. (in Russian)

[3] J. F. Sowa, "Conceptual graphs as a universal knowledge representation,” International journal computers \& mathematics with applications, vol. 23 (2-5), pp. 75-94, 1992.

[4] I. Niles and A. Pease, "Towards a standard upper ontology," in proceedings of the 2nd international conference on formal ontology and information systems (FOIS-2001), Ogunquit, 2001, pp. 2-9.

[5] V. B. Artemenko and A. A. Ivlev, "The ontology of military technologies based on conceptual maps,” Investigated in Russia, vol. 14, pp. 285-294, 2011. (in Russian)

[6] R. A. Calvo and J. Villalon, "Concept maps as cognitive visualizations of writing assignments,” Educational technology and society, vol. 14 (3), pp. 16-27, 2011. 
[7] I. M. Kobozeva and A. S. Marushkina, "An ontology of force interactions," in proceedings of the international scientific conference (Dialogue-2010) on computational linguistics and intelligent technologies, Bekasovo, 2010, vol. 9 (16), pp. 192199. (in Russian)

[8] G. B. Evgenev, B. V. Kuz'min, and A. A. Kokorev, "Development of intelligent CAD of technological processes," in collection of scientific papers on scientific session MIFI-2004, Moscow, 2004, vol. 3, pp. 43-44. (in Russian)

[9] V. Konevtsov, I. Poletaev, S. Verteshev, "Discrete automatic schemes for ASC TP,” in Environment. Technology. Resources: Proceedings of the 10th International Scientific and Practical Conference on Engineering sciences and production technologies, Rezekne, 2015, vol. I, pp. 67-71.

[10] P. C. Benjamin, C. P. Menzel, R. J. Mayer, F. Fillion, M. T. Futrell, P. S. deWitte, and M. Lingineni, "Information integration for concurrent engineering (IICE). IDEF5 Method Report," Knowledge based systems inc., Texas, USA. F33615C-90-0012, 21 Sep. 1994.

[11] T. A. Shiyan, "Formal ontology of material processes and modeling of global bio-social formations," Mathematical modeling of social processes, vol. 6, 2004. [Online serial]. Available:http://taras-shiyan.narod.ru/my mamosp/shiyan mamosp_6.pdf [Accessed: Feb. 25, 2021]. (in Russian)

[12] S. D. Danilova and N. B. Kim, "Application of the theory of processes in ontology with active semantics," in proceedings of the international scientific and practical conference on physics, mathematics and information technologies: actual problems, 2012. [Online]. Available: http://sibac.info/index.php/2009-0701-10-21-16/3127-2012-06-19-16-01-49. [Accessed: Feb. 25, 2021]. (in Russian)

[13] L. S. Bolotova, Yu. V. Moroz, S. S. Smirnov, and V. A. Smolyaninova, "The method of situational analysis and design of a domain model of arbitrary nature," in interuniversity collection of scientific papers on theoretical issues of computer technology and software, 2011. [Online]. Available: http://www.mtas.ru/bitrix/ components/bitrix/forum.interface/show file.php?fid=1937. [Accessed: Feb. 25, 2021]. (in Russian)

[14] V. F. Turchin, "The cybernetic ontology of actions," Kybernetes, vol. 22 (2), pp. 10-30, 1993

[15] S. Verteshev, V. Konevtsov, "Processes control with fuzzy initial information in a complex of software design of digital control systems," in Environment. Technology. Resources: Proceedings of the 11th International Scientific and Practical Conference on Engineering sciences and production technologies, Rezekne, 2017, vol. III, pp. 332-336.

[16] S. Verteshev, V. Konevtsov, "Direct digital control in a complex of software design of digital control systems,” in Environment. Technology. Resources: Proceedings of the 11th International Scientific and Practical Conference on Engineering sciences and production technologies, Rezekne, 2017, vol. III, pp. 337-342.

[17] G. K. Olkhovikov, "Towards new ontological premises of the logic of action: analysis of the L0 language,” Izvestiya of Ural
State University, Ser. 3, Social Sciences, No. 3 (69), pp. 5-17, 2009. (in Russian)

[18] D. A. Andreev, "Program for automated construction of a formalized description of the technology of the applied field of knowledge OntoTechnology," Certificate of state registration of computer programs No. 2013660420 the Russian Federation, 5 Nov., 2013. (in Russian)

[19] D. A. Andreev, Models, algorithms and indicators of quality of formalized description and analysis of production technologies, PhD in Technical Sciences [thesis]. Pskov: PSU, 2016.

[20] L. F. Pershina and S. V. Petrova, Technology of sewing production. Moscow: KDU, 2007. (in Russian)

[21] A. S. Statsenko, Technology of construction production. Russian Rostov-on-Don: Phoenix, 2006. (in Russian)

[22] B. M. Bazrov, Fundamentals of mechanical engineering technology. Moscow: Mashinostroenie, 2005. (in Russian)

[23] D. Andreev, S. Lyokhin, L. Motaylenko, and S. Verteshev, "Models and algorithms for constructing a formalized description of production technologies," in Environment. Technology. Resources: Proceedings of the 12th International Scientific and Practical Conference on Information Technologies, Rezekne, 2019, vol. II, pp. 21-27.

[24] D. A. Andreev, "Model of actions' concepts in ontological representations of technologies," in proceedings of the XXVII international scientific conference on mathematical methods in engineering and technologies, Tambov, 2014, vol. 3, pp. 85-87. (in Russian)

[25] D. A. Andreev and M. V. Voronov, "Method for constructing an ontology of technological actions," Bulletin of Saratov State Technical University, No. 3 (67), pp. 160-168, 2012. (in Russian)

[26] D. A. Andreev, "Modeling temporal relations in ontologies of technological actions,” Fundamental and applied problems of engineering and technology, No. 3 (299), pp. 40-49, 2013. (in Russian)

[27] D. A. Andreev and M. V. Voronov, "Algorithm for constructing a decomposition structure of technology of the applied field of knowledge," in abstracts of the VIII international conference on mathematical modeling in education, science and production, Tiraspol, 2013, pp. 6-8. (in Russian)

[28] I. Antonov, I. Bruttan, D. Andreev, and L. Motaylenko, "The method of automated building of domain ontology," in Environment. Technology. Resources: Proceedings of the 12th International Scientific and Practical Conference on Information Technologies, Rezekne, 2019, vol. II, pp. 34-37.

[29] D. A. Andreev and I. V. Antonov, "System-ontological approach to machine description of the component environment of technological processes,” Industrial ACS and controllers, No. 9, pp. 29-34, 2012. (in Russian)

[30] D. Andreev, S. Lyokhin, V. Nikolaev, and O. Poletaeva, "Development of software for design ontological representations of production technologies," in Environment. Technology. Resources: Proceedings of the 12th International Scientific and Practical Conference on Information Technologies, Rezekne, 2019, vol. II, pp. 28-33. 